\title{
Lensing \& Dynamics in the Galaxy Cluster MS2137-23
}

\author{
R. Gavazzi \\ Institut d'Astrophysique de Paris, UMR 7095, 98bis Boulevard Arago, 75014 Paris, France, \\ email: gavazzi@iap.fr
}

\begin{abstract}
We present a new model of the lensing cluster of galaxies MS2137-23. By incorporating strong and weak lensing data in a fully elliptical lens modeling, we show that the total density profile must be close to the predictions of numerical simulations with an inner slope well consistent with a NFW profile. The model that best fits the lensing constraints is used to infer to line-of-sight velocity distribution (LOSVD) of stars in the central $\mathrm{cD}$ galaxy. This distribution is found to be far from Maxwellian. The important non-Gaussian tails produce a significant low-bias when measuring the velocity dispersion by assuming Gaussian absorption lines. In the case of MS2137, most of the information comes from multiples arcs. Internal kinematics of stars only help modeling the innermost kiloparsecs. However, in clusters with fewer constraints (no radial arcs for instance), a detailed modeling of both strong lensing and internal kinematics is crucial.
\end{abstract}

\section{Introduction}

N-body numerical simulations actually provide the main theoretical way of studying the CDM paradigm at the very small scales. Precisely for this reason, it is important to test those conclusions that strongly depend on the nature of dark matter well. Three small scale observations act as key-tests for CDM : the density profile of dark matter halos (and especially its inner parts), the abundance of substructures within main halos and the triaxiality of halos. This note focuses on the former issue. Most CDM simulations predict a universal profile of the general form :

$$
\rho\left(r / r_{s}\right)=\rho_{s}\left(r / r_{s}\right)^{-\alpha}\left(1+r / r_{s}\right)^{\alpha-3},
$$

with an inner slope $\alpha$ ranging between $\alpha=1$ (NFW) and $\alpha=1.5$ (Navarro et al. 1997; Moore et al. 1998; Ghigna et al. 2000; Jing \& Suto 2000).

The rotation curves of low surface brightness galaxies are often seen as a direct evidence that CDM simulations fail at reproducing very small scales (e.g. de Blok \& Bosma 2002, and references therein). These observations suggest a flatter dark matter density profile $\frac{\mathrm{d} \ln \rho}{\mathrm{d} \ln r}=-0.2 \pm 0.2$ for $r \lesssim 1 \mathrm{kpc}$. This apparent contradiction with simulations is one of the principal arguments for the so-called cusp-core debate.

Except below a few tens of kpc, the dark matter halo is known to dominate the total mass budget of clusters, as in dwarfs/LSB galaxies. The inner density profile of clusters thus provides a valuable and complementary test for the existence of a universal density profile. The controversy has recently been extended to clusters of galaxies. The recent analysis of Sand et al. (2002, 2004, hereafter Sa0204) suggests that the inner parts of clusters of galaxies may not be consistent with simulations. By modeling the kinematics of stars in the central cD galaxy of six clusters and their critical lines, Sa0204 found that the inner density slope must be as shallow as $\alpha \sim 0.52$ for the subsample with radial arcs (including MS2137-23) and $\alpha<0.57$ for those clusters without a radial arc. More 
recently, Dalal \& Keeton (2003) and Bartelmann \& Meneghetti (2003) have contested the strong lensing approach of Sa0204 because these authors had neglected the lens ellipticity.

In this note, we revisit the radial mass profile of the cluster MS2137 because of its outstanding lens properties. This analysis builds on the previous work of Gavazzi et al. (2003, hereafter, G03). We describe the lens modeling in section 2 and show that strong and weak lensing constraints can be well modeled by a NFW profile between $10 \mathrm{kpc}$ and $1 \mathrm{Mpc}$. In section 3 , we focus on the internal dynamics of the central cD galaxy. The aim of this section is to fully derive the line-of-sight velocity distribution LOSVD as a function of projected radius in order to demonstrate that it is not Gaussian. We discuss how important is this bias for constraining the radial mass profile by coupling the velocity dispersion profile to lensing data. Finally, section 4 discusses the origin of the discrepancy between our results and those of Sa0204 and concludes. Except otherwise stated, we assume a $\Omega_{m}=0.3, \Omega_{\Lambda}=0.7$ and $h=0.7$ concordance cosmology.

\section{Lens modeling}

In this section we focus on the density profile modeling using lensing constraints only. The lens properties of the cluster have been extensively studied (Fort et al. 1992; Mellier et al. 1993; Miralda-Escudé 1995; Bartelmann 1996; Hammer et al. 1997; Gavazzi et al. 2003). The cluster lies at $z_{l}=0.313$ and the sources responsible of the radial and tangential arcs both lie at $z_{s} \approx 1.501$, leading to the critical surface density $\Sigma_{\text {crit }}=2.39 \times 10^{9} h_{70} \mathrm{M}_{\odot} \mathrm{kpc}^{-2}$. We use the 26 multiple conjugate knots in the tangential and radial systems as discussed in G03. We exclude the central core image candidate since its detection is marginal. We developped our own ray-tracing inversion software by implementing many features of lensmodel (Keeton 2001). The strong lensing modeling is based on a source plane $\chi_{\mathrm{src}}^{2}$ minimization.

In addition, we simultaneously include weak lensing data (also presented in G03). The catalogue of background "weakly lensed" galaxies comes from VLT/FORS and VLT/ISAAC images for which we were able to derive a good estimate of photometric redshifts using $U B V R I J K$ bands. Instead of fitting a parameterized model on $\left\langle e_{t}\right\rangle(\theta)$ measurements, we fully compute the likelihood as a function of model parameters (e.g. Schneider et al. 2000):

$$
\mathcal{L}_{\mathrm{wl}}=\prod_{i=1}^{N_{\mathrm{bg}}} p_{s}\left(e_{s}\left(e_{i}, z_{i}\right)\right)\left|\frac{\mathrm{d} e_{s}}{\mathrm{~d} e_{i}}\right|,
$$

where $e_{i}$ is the observed ellipticity and $e_{s}$ the intrinsic ellipticity of the background source galaxy. For small reduced shear $g$, the relation between $e_{i}$ and $e_{s}$ reads : $e_{i} \approx e_{s}+g$.

We model the lens potential with two components : the stellar content of the central cD galaxy and the cluster dark matter halo with either a NFW profile or a cusp profile (see below). The $\mathrm{cD}$ galaxy is described by an Hernquist profile of the form :

$$
\rho_{*}(r)=\frac{\rho_{s *} r_{s *}^{4}}{r\left(r_{s *}+r\right)^{3}},
$$

which turns out to provide a better fit than a Jaffe profile. The stellar scale radius is $r_{s *}=11.1 \pm 2 h_{70}^{-1} \mathrm{kpc}$. The rest frame $V$ band luminosity is $L_{V}=4.77 \pm 0.4 \times 10^{11} h_{70}^{-2} L_{\odot}$ and the stellar mass is $M_{*}=2 \pi \rho_{s *} r_{s *}^{3} \equiv \Upsilon_{V} L_{V}$ where $\Upsilon_{V}$ is the rest-frame $\mathrm{V}$ band stellar mass-to-light ratio. $\Upsilon_{V}$ (or $M_{*}$ ) is the only free parameter for the stellar component. The 


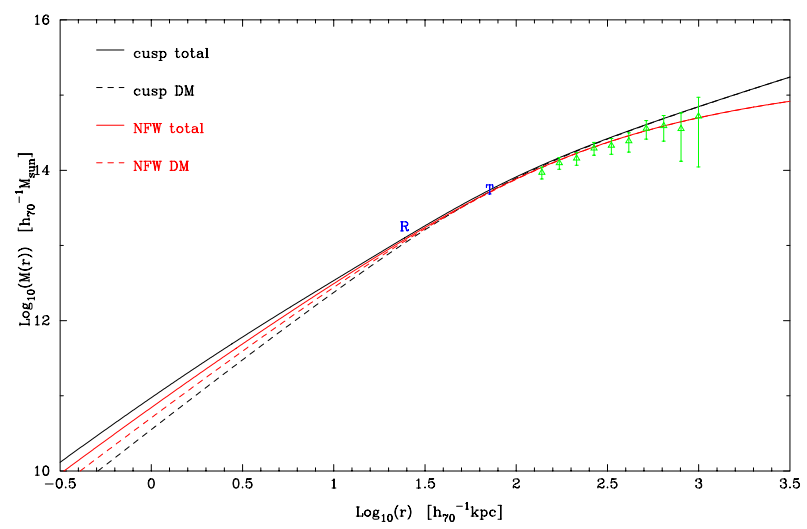

Figure 1. Projected mass profile of models "cusp" (black) and NFW (red). Solid line : total mass profile and dashed line : contribution of dark matter. The radial (R) and tangential $(\mathrm{T})$ arcs location are plotted. The inferred profiles are very similar over the region probed by arcs i.e. from 20 to $200 \mathrm{kpc}$. Green error bars show the $\zeta$-statistic from weak lensing data.

dark matter halo profile reads :

$$
\rho(r)=\left\{\begin{array}{lr}
\rho_{s} x^{-1}(1+x)^{-2} & \mathrm{NFW} \\
\rho_{s} x^{-\alpha}\left(1+x^{2}\right)^{(\alpha-\beta) / 2} & \text { cusp }
\end{array},\right.
$$

with $x=r / r_{s}$ and $r_{s}$ the dark matter scale radius. The cusp model slightly differs of the form (1.1) but numerical integrations are much easier (Chae 2002). It has a faster transition between the inner slope $\alpha$ and the outer slope $\beta$, which is left as a free parameter. All the models components are elliptical. The orientation and axis ratio of stars are settled from the observed light distribution whereas those of dark matter are treated as free parameters. Consequently, the cusp model has 7 free parameters : dark matter scale radius $r_{s}$, dark matter virial mass $M_{200}$, the inner and outer slopes $\alpha$ and $\beta$, the position angle $P A$ and axis ratio $q$ of the dark matter halo and the stellar mass $M_{*}$, whereas the NFW model only has 5 free parameters since $\alpha$ and $\beta$ are set to the values 1 and 3 respectively.

The most interesting best fit model parameters and their corresponding 95\% CL errors are:

- cusp: $r_{s}=54.7_{-9.7}^{+17.0} h_{70}^{-1} \mathrm{kpc}, q=0.765 \pm 0.007, \alpha=0.88_{-0.09}^{+0.13}, \beta=2.26_{-0.14}^{+0.22}$ and $M_{*}=3.4 \pm 0.6 \times 10^{12} h_{70}^{-1} \mathrm{M}_{\odot}$ with $\chi^{2} /$ dof $=0.9 ;$

- NFW: concentration parameter $c=9.9 \pm 0.5$, virial mass $M_{200}=2.2_{-0.3}^{+0.4} \times 10^{14} h_{70}^{-1} \mathrm{M}_{\odot}$, virial radius $r_{200}=1240_{-60}^{+68} h_{70}^{-1} \mathrm{kpc}$, potential axis ratio $q=0.899 \pm 0.004$ and $M_{*}=$ $1.1_{-0.4}^{+0.3} \times 10^{12} h_{70}^{-1} \mathrm{M}_{\odot}$ with $\chi^{2} /$ dof $=1.2$.

The projected total and dark matter mass profiles of each model are plotted on Fig. 1. One can see a remarkable agreement between profiles for $10 h_{70}^{-1} \mathrm{kpc} \lesssim r \lesssim 1 h_{70}^{-1} \mathrm{Mpc}$. Weak lensing data are too noisy and do not extend far enough to constrain the outer parts of the density profile but show that strong lensing models can safely be extrapolated up to the virial radius $r_{200} \sim 1.2 h_{70}^{-1} \mathrm{Mpc}$. Most discrepancies between the cusp and NFW models occur above this radius and below $10 h_{70}^{-1} \mathrm{kpc}$. These latter scales can be probed by internal kinematics of star in the $\mathrm{cD}$ galaxy as proposed by Miralda-Escudé (1995) and applied by Sa0204. We show in the next section that such a technique requires a careful dynamical analysis. 


\section{Dynamics of stars in the central $\mathrm{cD}$ galaxy}

The usual way to derive the mass profile from measurements of stellar kinematics in galaxies is based on the Jeans equation that relates the density of tracers $\nu(r)$, the total mass profile $M_{\mathrm{tot}}(r)$, the second order moment of the radial velocity $\overline{v_{r}^{2}}$ and the anisotropy parameter $\eta(r)=1-\frac{\overline{v_{t}^{2}}}{v_{r}^{2}}$ through :

$$
\frac{1}{\nu} \frac{\mathrm{d}\left(\nu \overline{v_{r}^{2}}\right)}{\mathrm{d} r}+2 \frac{\eta \overline{v_{r}^{2}}}{r}=-\frac{G M_{\mathrm{tot}}}{r^{2}} .
$$

To be compared to observations, this relation must be projected along the line-of-sight in order to express the projected line-of-sight velocity dispersion $\sigma_{\text {los }}$ as a function of the projected radius $R$. It is generally assumed that the underlying line-of-sight velocity distribution (LOSVD) is Gaussian. Using Osipkov-Merritt dynamical models (Osipkov 1979; Merritt 1985), we show in the following that this assumption is erroneous and leads to a biased velocity dispersion profile (see also Kazantzidis et al. 2004).

From the reduced potential $\Psi(r)$, the stellar mass density $\rho_{*}(r)$ and the quantity

$$
Q=\Psi(r)-\frac{v^{2}}{2}\left[1+\frac{r^{2}}{r_{a}^{2}} \sin ^{2} \zeta\right]
$$

one can derive the distribution function of $Q$ using the Eddington formula (Binney \& Tremaine 1987) :

$$
\tilde{f}(Q)=\frac{1}{\sqrt{8} \pi^{2}}\left[\int_{0}^{Q} \frac{\mathrm{d}^{2} \tilde{\rho}_{*}}{\mathrm{~d} \Psi^{2}} \frac{\mathrm{d} \Psi}{\sqrt{Q-\Psi}}+\frac{1}{\sqrt{Q}}\left(\frac{\mathrm{d} \tilde{\rho}_{*}}{\mathrm{~d} \Psi}\right)_{\Psi=0}\right] .
$$

This model reduces to an isotropic tensor of velocities when the anisotropy radius $r_{a} \rightarrow$ $\infty$. In this case, $Q \rightarrow \mathcal{E}=\Psi(r)-\frac{v^{2}}{2}$. $\zeta$ is the polar angle between the velocity $\mathbf{v}$ and $\mathbf{r}$ and $\tilde{\rho}_{*}=\left(1+\frac{r^{2}}{r_{a}^{2}}\right) \rho_{*}$. We can formally integrate the relation (3.3) along the line-of-sight coordinate $(z)$ and perpendicular velocities $v_{\perp}$ to derive the LOSVD:

$$
p\left(R, v_{\text {॥ }}\right)=2 \pi \int_{v_{\|}^{2}}^{2 \Psi(R)} \mathrm{d} v^{2} \int_{0}^{z_{m}(v)} \mathrm{d} z \int_{-1}^{1} \mathrm{~d}(\cos \zeta) f(Q) .
$$

$z_{m}(v)$ is the maximum value of $z$ satisfying $2 \Psi\left(\sqrt{R^{2}+z_{m}^{2}}\right)=v^{2}$, i.e. for which stars are bound to the system. The integration of eq (3.4) is time-consuming especially if one accounts for the observational smearing by the PSF and the finite slit width. Consequently, we have implemented a Monte-Carlo integration technique that fully handles these observational features and allows to derive the $\operatorname{LOSVD} p\left(R, v_{11}\right)$. It is then straighforward to infer the line-of-sight velocity dispersion $\sigma_{\text {los }}(R)$ and the kurtosis $\kappa={\overline{v_{11}^{4}}}_{{\overline{v^{2}}}^{2}}^{2}-3$.

When applied to the best fit lensing models of $\mathrm{MS} 2137, p\left(R, v_{11}\right)$ turns out to be far from Gaussian. Hence, velocity dispersion measurements of Sa0204 are likely to be significantly biased. This low-biased quantity will be referred to as $\sigma_{h_{4}}(R)$. van der Marel \& Franx (1993) have shown that

$$
\sigma_{h_{4}}(R) \simeq \sigma_{\operatorname{los}}(R) \frac{1}{1+\kappa(R) / 8} .
$$

Figure 2 shows the radial profiles $\sigma_{h_{4}}(R)$ and $\sigma_{\text {los }}(R)$ for the NFW and cusp mass profiles of section 2 and an isotropic velocity tensor. The curves thickness is characteristic of the scatter in parameter estimate from lens modeling showing that strong lensing provides very tight constraints. Sa0204 data are also displayed as red boxes. Without fitting, 


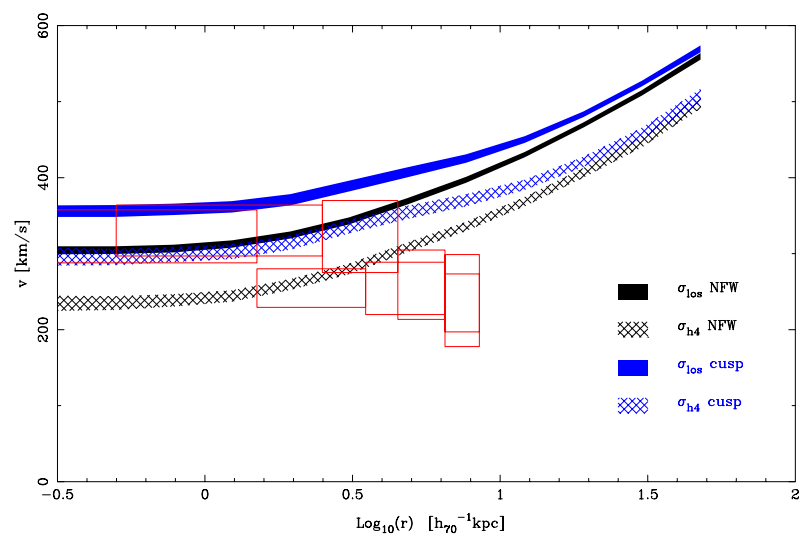

Figure 2. Velocity profiles for isotropic orbits. The NFW (resp. cusp) profile is black (resp. blue). $\sigma_{\text {los }}(R)$ (full filling) and $\sigma_{h_{4}}(R)$ (hatched filling) differ by 20 to $45 \%$ showing that $p\left(R, v_{॥}\right)$ is not Gaussian. The red boxes are velocity measurements of Sa0204. The curves thickness is characteristic of the scatter in parameter estimates from lens modeling.

the lensing-based models are fairly consistent with velocity measurements. Furthermore, fitting velocity data will clearly give different results with $\sigma_{\operatorname{los}}$ or $\sigma_{h_{4}}$.

\section{Discussion \& Conclusion}

Using joined strong and weak lensing constraints in the cluster of galaxies MS2137-23, we have shown that the radial dark matter density profile is consistent with the NFW model. X-rays measurements of Allen et al. (2001) confirm our conclusions. Small departs may occur at very small scales $r \lesssim 1 \mathrm{kpc}$, which are dominated by stars and are well below the actual spatial resolution of numerical simulations.

We can advocate three main reasons why Sa0204 found the inner slope to be shallower than NFW. With decreasing relevance : they first neglected the lens ellipticity, they fixed the cluster scale radius to a high value (i.e. low concentration) and they assumed a Gaussian LOSVD, then underestimating the true velocity dispersion.

A detailed modeling of both radial and tangential arcs (including their size, shape, location and that of their counter-images) provides very tight constraints and is not much sensitive to the velocity bias we have highlighted in this note. Nevertheless, coupling dynamics and lensing on clusters with fewer arcs is likely to suffer from this bias and requires a particular attention in the dynamical modeling.

\section{Acknowledgements}

I would like to acknowledge helpful discussions with J. Miralda-Escudé, B. Fort, Y. Mellier and G. Mamon. I am also thankful to D. Sand who kindly made available velocity dispersion data.

\section{References}

Allen, S. W., Schmidt, R. W., \& Fabian, A. C. 2001, MNRAS, 328, L37

Bartelmann, M. 1996, A\&A, 313, 697

Bartelmann, M. \& Meneghetti, M. 2003, astro-ph/0312011

Binney, J. \& Tremaine, S. 1987, Galactic dynamics (Princeton University Press, 1987)

Chae, K. 2002, ApJ, 568, 500

Dalal, N. \& Keeton, C. R. 2003, astro-ph/0312072 
de Blok, W. J. G. \& Bosma, A. 2002, A\&A, 385, 816

Fort, B., Le Fevre, O., Hammer, F., \& Cailloux, M. 1992, ApJ, 399, L125

Gavazzi, R., Fort, B., Mellier, Y., Pelló, R., \& Dantel-Fort, M. 2003, A\&A, 403, 11

Ghigna, S., Moore, B., Governato, F., et al. 2000, ApJ, 544, 616

Hammer, F., Gioia, I. M., Shaya, E. J., et al. 1997, ApJ, 491, 477

Jing, Y. P. \& Suto, Y. 2000, ApJ, 529, L69

Kazantzidis, S., Magorrian, J., \& Moore, B. 2004, ApJ, 601, 37

Keeton, C. 2001, astro-ph/0102340

Mellier, Y., Fort, B., \& Kneib, J. 1993, ApJ, 407, 33

Merritt, D. 1985, AJ, 90, 1027

Miralda-Escudé, J. 1995, ApJ, 438, 514

Moore, B., Governato, F., Quinn, T., Stadel, J., \& Lake, G. 1998, ApJ, 499, L5+

Navarro, J. F., Frenk, C. S., \& White, S. D. M. 1997, ApJ, 490, 493

Osipkov, L. P. 1979, Pis ma Astronomicheskii Zhurnal, 5, 77

Sand, D. J., Treu, T., \& Ellis, R. S. 2002, ApJ, 574, L129

Sand, D. J., Treu, T., Smith, G. P., \& Ellis, R. S. 2004, ApJ, 604, 88

Schneider, P., King, L., \& Erben, T. 2000, A\&A, 353, 41

van der Marel, R. P. \& Franx, M. 1993, ApJ, 407, 525 